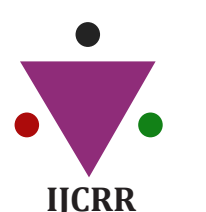

ection: Healthcare

Sci. Journal Impact

Factor: 6.1 (2018)

ICV: 90.90 (2018)

(c) (i) (3)

Copyright@IJCRR

\title{
Radiological Findings of Spinal Neurocysticercosis
}

\section{Vasant Gawande ${ }^{1}$, Kunal Saoji ${ }^{2}$, Ajith Nair ${ }^{3}$, Kiran Saoji ${ }^{4}$}

'Associate Professor, Department of Orthopedics Jawaharlal Nehru Medical College, Datta Meghe Institute of Medical Sciences, Sawangi (Meghe), Wardha, MS, India; ${ }^{2}$ Assistant Professor, Department of Orthopedics Datta Meghe Medical College, Shalinitai Meghe Hospital and Research Centre, Nagpur, MS, India; 3]unior Resident Department of Orthopedics Jawaharlal Nehru Medical College, Datta Meghe Institute of Medical Sciences, Sawangi (Meghe), Wardha, MS, India; 4 Professor, Department of Orthopedics Jawaharlal Nehru Medical College, Datta Meghe Institute of Medical Sciences, Sawangi (Meghe), Wardha, MS, India.

\section{ABSTRACT}

Introduction: Neurocysticercosis is one of the most common parasitic diseases of the CNS in which the central nervous system is involved by taenia solium. However, it is uncommon that neurocysticercosis involves the spine.

Result: Here, we reported a 35-year-old man with intramedullary cysticercosis in the thoracic spinal cord. MRI of the spine revealed a small $(0.5 \times 0.7 \mathrm{~cm})$ intramedullary lesion in the cord at the level of D8 vertebral body.

Conclusion: Since the patient had progressive neurological deficits, surgery was recommended to decompress the spinal cord. Here, we also conversed the diagnosis and management of intramedullary cysticercosis in the combination of the literature review.

Key Words: Intramedullary, Cysticercosis, Spinal cord

\section{INTRODUCTION}

Cysticercosis is the commonest parasitic disease to affect the central nervous system (CNS). According to the review of published literature, more than 2.5 million people worldwide are infected. ${ }^{1}$

Encysted larval form of Taenia solium (Cysticerus cellulose) commonly called porcine tapeworm causes neurocysticercosis. The parenchyma of brain, intracranial subarachnoid space and ventricular system is involved when it affects the central nervous system.

Intraspinal cord involvement is reported in only 1 to $5 \%$ of patients, of which leptomeningeal involvement is more common than intramedullary involvement.

Neurocysticercosis is one of the most common parasitic diseases of the CNS in which the central nervous system is involved by taenia solium. However, it is uncommon that neurocysticercosis involves the spine accounting for $1.2 \%$ to $5.8 \%$ of all cases of neurocysticercosis.

Based on the location of cysticercus in the spine, Cysticercosis has been classified anatomically as extraspinal (vertebral) or intraspinal (epidural, subdural, arachnoid, or intramedullary), among which intramedullary type is very rare.

\section{CASE}

A 35-year-old man presented with 3 episodes of seizures over 1 month. It was followed by trouble in walking associated with falls which were followed by severe weakness in both lower limbs since 15 days making him confined to bed. His bladder and bowel habits were normal. He also complained of abnormal sensations like pins and needles in both lower limbs.

On examination, his spine was normal. The patient had spasticity and power of grade $2 / 5$ in the lower limbs, wasting of lower limb muscles with hypoesthesia below T7 level, and exaggerated deep tendon reflexes in his lower limbs. Both Planters were extensors.

With these features, the patient was referred to the radiology department.

\section{Imaging}

MRI of the spine revealed a small $(0.5 \times 0.7 \mathrm{~cm})$

\section{Corresponding Author:}

Dr. Kunal Saoji, Assistant Professor, Department of Orthopedics, Datta Meghe Medical College, Shalinitai Meghe Hospital and Research Centre, Nagpur, MS, India.

ISSN: 2231-2196 (Print)

Received: 22.07 .2020
ISSN: 0975-5241 (Online)

Revised: 25.08.2020
Accepted: 5.09 .2020
Published: 22.09 .2020 
intramedullary lesion in the cord at the level of D8 vertebral body.

On T1weighted image, it was isointense to CSF and on T2 it was slightly hypointense to CSF. Hyperintensity was noted in FLAIR. There was e/o a hypointensity around the lesion on T2 images suggestive of minimal oedema. Post-contrast MRI showed enhancement in the area ( Fig 1 and 2)

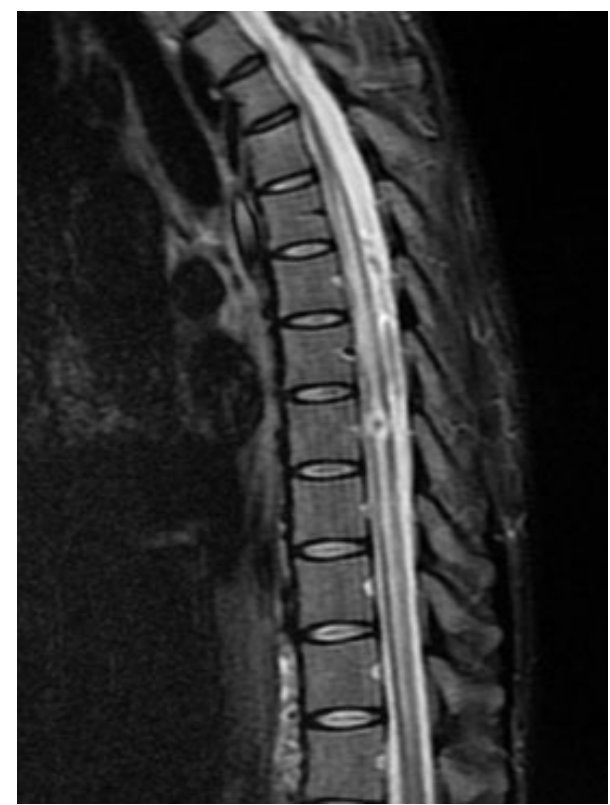

Figure 1: MRI T2 weighted image showing a ring-like lesion appearing hypo intense to CSF in the intramedullary compartment of thoracic cord.

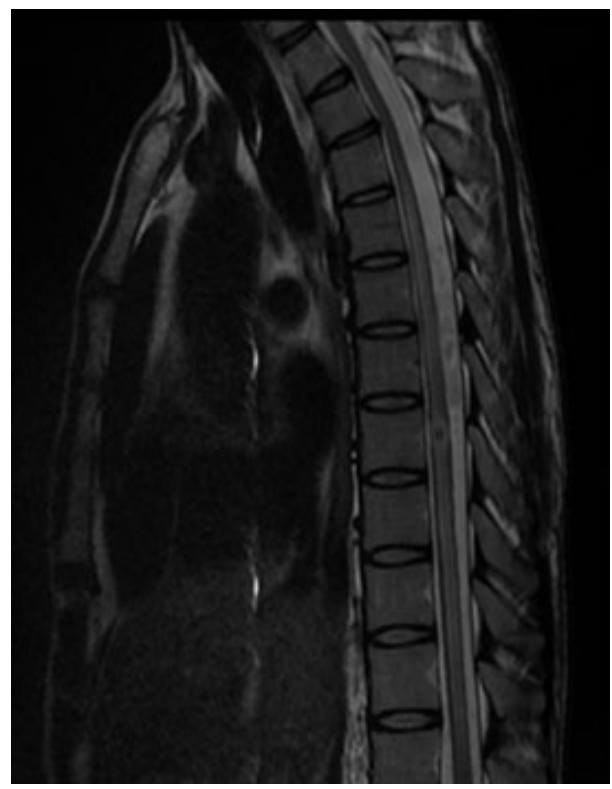

Figure 2: MRI FLAIR showing a ring like lesion appearing hyper intense to CSF in the intramedullary compartment of thoracic cord.

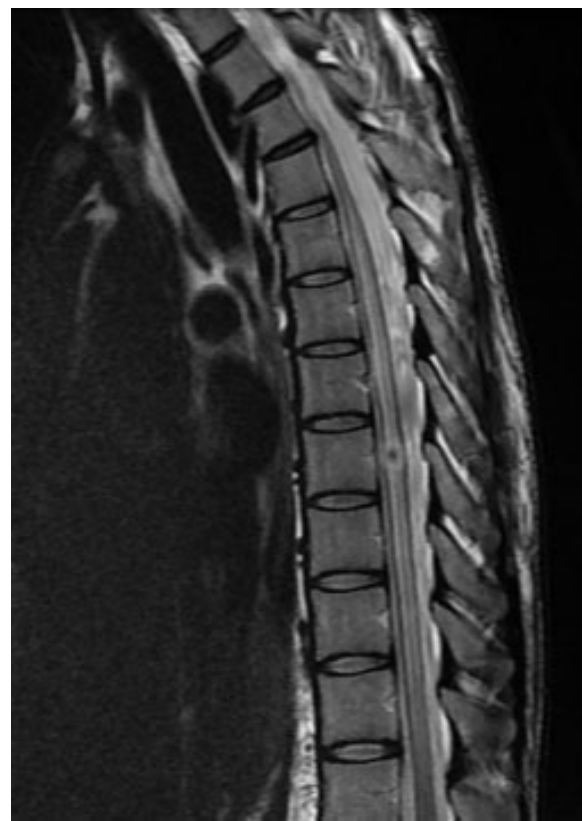

Figure 3: Contrast enhanced MRI shows intensely enhancing rim like lesion in the intramedullary compartment of thoracic cord.

With this presentation, possibility of an infective aetiology such as Tuberculoma or Neurocysticercosis was considered. The MRI of the brain was performed, which revealed multiple small ring enhancing lesions with hole with dot appearance in few, thus confirming diagnosis of Neurocysticercosis ( Fig 3).

\section{DISCUSSION}

Spinal neurocysticercosis is commonly encountered in the CSF spaces surrounding the cord. This site is common in spinal neurocysticercosis because of dissemination of lesions from brain via CSF. ${ }^{2}$

Intramedullary NCC is an extremely rare condition. The probable etiology is hematogenous dissemination from a primary source other than CNS (Fig no 1). Thoracic spine is the preferred site for intramedullary NCC due to higher blood volume in this area as compared to other spinal segments ${ }^{3}$. In present case also thoracic involvement in noted.

MR imaging of intact intramedullary cysts typically shows cystic areas within the spinal cord along with intensity of cyst fluid that is analogous to CSF on both TI- and T2- (Fig no 2) weighted images. Sometimes the scolex can be recognized as a mural nodule within the cavity of cyst on TIweighted images.

However it was not seen in the cord in our case but it was seen in the sections of brain. As in the brain, degenerating cysticercotic cysts within the spinal cord may show a subtle hypointense rim surrounding the intramedullary cyst on 
T2-weighted images and evidence of irregular peripheral enhancement after IV gadolinium administration. ${ }^{3}$ Similar finding were found in present case also.

The differential diagnosis of a small ring enhancing area in the cord will be tuberculoma, metastasis and abscess. The closest differential diagnosis is tuberculoma, however tuberculoma will usually have a size of $2-8 \mathrm{~mm}$ and appear hypoor isointensity or central hyperintensity with a hypointense rim on T2W images and isointensity and/or hypointensity on T1W images (Fig no 3 ). ${ }^{4}$

The other differential diagnosis includes cerebral metastasis and abscess. However cerebral metastasis appear isointense to hypointense on $\mathrm{T} 1 \mathrm{~W}$ images and typically hyperintense on T2W images. The enhancement pattern in it can be uniform, punctate, or ring-enhancing, but it is usually intense. ${ }^{5}$

Abscess appears hypointense on T1W images and hyperintense on T2, but there is surrounding vasogenic edema, on T2W images it appears as surrounding hyperintensity. With iv contrast abscess shows typical rim like enhancing pattern. 6

The CSF examination frequently shows raised proteins, a low or normal glucose level, moderate level of lymphocytic pleocytosis and eosinophilia. ELISA or serum enzymelinked immunoelectric transfer bolt assay helps in finding cysticercal antibodies in CSF and have good sensitivity and specificity in cysticercosis diagnosis. ${ }^{7}$

Timely diagnosis and management can increase the result. Sharma reported that $60 \%$ patients acquired improvement after surgery, $25 \%$ did not improve, and $15 \%$ died $^{3}$. In recent years studies, there is significant increase in results by surgical management; there is no death and most of patients may possibly live devoid of special support. When in doubt surgical management is the management of choice or else medical therapy has its benefits. ${ }^{8,9}$

Albendazole is a drug which is effective since 1996 in patients with intramedullary cysticercosis. It is thought that preoperative adjunctive treatment with albendazole helps to stabilize the lesion and thus causes a strong dissection plane during surgery. Albendazole is generally used postsurgery as a routine therapy $(15 \mathrm{mg} / \mathrm{kg} /$ day $)$ for 4 to 6 weeks, according to the idea that cysticercosis is a generalized disease with focal manifestation. Moreover, Albendazole is often combined with corticosteroids, for the reason that its level in the blood can increase by the latter. ${ }^{9,10}$

\section{CONCLUSION}

To conclude we suggest that neurocysticercosis should be considered in the differential diagnosis of ring lesions involving the cord, particularly in endemic areas.

\section{REFERENCES}

1. Bin Qi, Pengfei Ge, Hongfa Yang, Chunhua Bi, and Yiping Li, Spinal Intramedullary Cysticercosis: A Case Report and Literature Review, Int Journal of Medical Science. 2011; 8(5): 420-423.

2. Eric T. Kimura-Hayama, MD, Jesús A. Higuera, MD, Roberto Corona-Cedillo, MD, et al Neurocysticercosis: Radiologic-Pathologic Correlation, radiographocs; 2010; 30(6);1705-1707

3. Claudia C. Leite1 J. Randy Jinkins1 Beatriz E. Escobar1 Alvaro C. Magalhaes 2 et al ,MR Imaging of Intramedullary and Intradural-Extramedullary Spinal Cysticercosis, AJR:169, December 1997; 1713-1717

4. Sheth TN, Pillon L, Keystone J et-al. Persistent MR contrast enhancement of calcified neurocysticercosis lesions. AJNR Am J Neuroradiol. 1998;19 (1): 79-82

5. Eichler AF, Loeffler JS. Multidisciplinary management of brain metastases. Oncologist. 2007;12 (7): 884-98.

6. Haimes AB, Zimmerman RD, Morgello S. et al. MR imaging of brain abscesses. AJR Am J Roentgenol. 1989;152 (5): 1073-85

7. Tsang VC, Brand JA, Boyer AE. An enzyme-linked immunoelectro transfer blot assay and glycoprotein antigens for diagnosing human cysticercosis. J Infect Dis. 1989;159(1):50-9.

8. Kasliwal MK, Gupta DK, Suri V. et al. Isolated spinal neurocysticercosis with clinical pleomorphism. Turkish Neurosurgery. 2008;18(3):294-7.

9. Garg RK, Nag D. Intramedullary spinal cysticercosis: response to albendazole: case reports and review of the literature. Spinal Cord. 1998;36(1):67-70.

10. Corral I, Quereda C, Moreno A. et al. Intramedullary cysticercosis cured with drug treatment. A case report. Spine. 1996;21(19):2284-7. 